\title{
An impressive portfolio of product innovations
}

Under the banner of 'Smile for the World Since 1921 - 100 years of Quality in Dental, GC held an international press conference on 20 September to introduce GC's latest cutting edge developments being featured at IDS 2021 in Cologne.

This year's IDS has been of particular significance to the company as it took place during GC's 100th anniversary year. Unlike in previous years, GC felt it was their corporate responsibility in the current climate to organise this special press conference online.

With a strong focus on how GC can support and empower today's clinicians and technicians, Josef Richter, COO \& President, GC Europe outlined a selection of GC's priority products. These included:

- EQUIA Forte HT - the new bulk fill glass fill hybrid restorative system that needs no complex finishing or polishing, achieving a finished restoration in just 3.5 minutes

- G-ænial A'CHORD - the new universal composite featuring exceptional technology with a simple unishade system
- G2-BOND Universal - a new standard of universal bonding with the convenient choice of self-etch and etch-and-rinse in one system for a multiple range of indications

- G-CEM ONE - the new self-adhesive resin cement with self-curing ability for a wide range of indications in just one syringe

- GC Initial LiSi Block - the first lithium disilicate CAD/CAM block that does not require crystallisation firing ensuring smooth, accurate margins, durable aesthetics and predictable outcomes

- GC Initial IQ ONE SQIN - the new and highly aesthetic, paintable colour-andform ceramic system for the creation of all zirconia and lithium disilicate restorations.

For more information on GC UK's restorative portfolio contact GC UK Ltd on 01908 218999, email info.uk@gc.dental or visit https://europe.gc.dental/en-GB/ products/restore.

\section{The latest one-appointment CAD/CAM chairside option}

With its High Density Micronisation (HDM) CAD/CAM technology, Initial LiSi Block is a fully crystallised lithium disilicate block ready to be polished right after milling and without firing.

Initial LiSi Block's unique ultrafine crystal makes it easy to grind and can be quickly milled in its fully crystallised state. Thanks to Initial LiSi Block's exceptional properties, high gloss and natural opalescence can be achieved in just a few minutes by polishing only, with the restoration then ready for luting.

Initial LiSi Block is available in four aesthetic shades and two translucencies. When further characterisation is required, the Initial ceramic product portfolio offers
Lustre Pastes ONE and Spectrum Stains. These fully synergistic aesthetic solutions means the chroma, value and brightness can be easily adjusted and more depth and detail can be added just by painting.

\section{Initial LiSi Block is the first lithium} disilicate CAD/CAM block that does not require crystallisation firing, ensuring smooth, accurate margins, durable aesthetics and predictable outcomes the ideal one-appointment CAD/CAM chairside option for today's clinicians.

For more information about GC Initial LiSi Block and GC's restorative portfolio contact GC UK on 01908 218999, email info.uk@gc.dental or visit https://europe. gc.dental/en-GB/products/initiallisiblock.
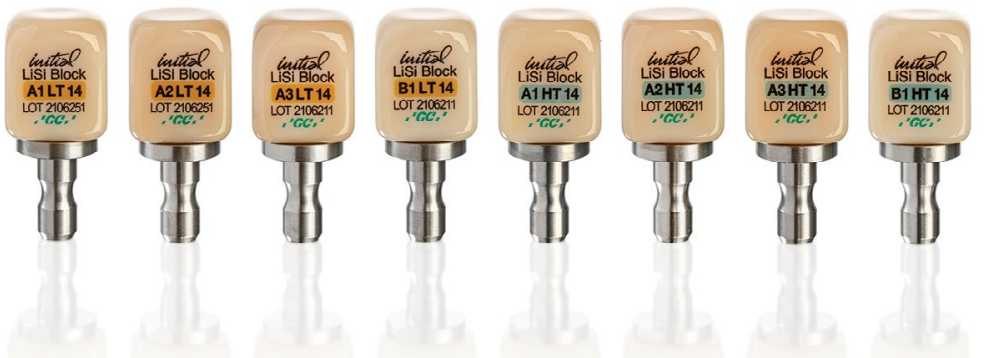

\section{A unique approach to bone regeneration}

The OSSIX range of bone regeneration materials, using GLYMATRIX core technology, is designed for clinical flexibility and ease of use, providing the ability to solve common regenerative challenges to achieve the best clinical outcomes.

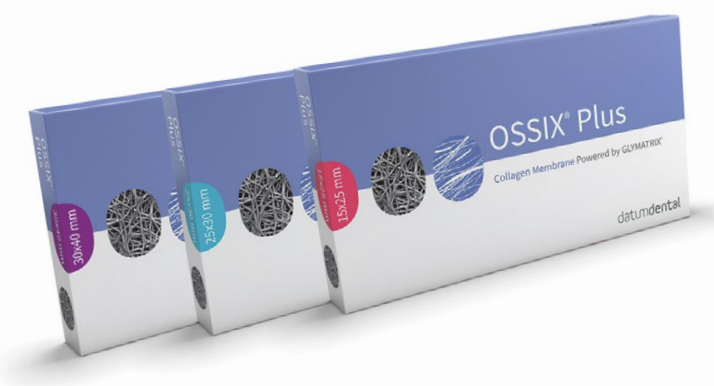

OSSIX PLUS - resilient, resorbable collagen membrane:

- Maintains barrier functionality for 4-6 months

- Resists degradation when exposed for 3-5 weeks

- Offers excellent handling properties, adapts and conforms to defects and adheres well to tissue.

OSSIX VOLUMAX - thick collagen regenerative matrix:

- Contains the same GLYMATRIX technology as OSSIX PLUS

- Thick and expands when wet

- Excellent handling, adapts and adheres to bone.

OSSIX Bone - a sponge-like ossifying block for true bone formation:

- For use in intraosseous defects, socket preservation and internal sinus lifts

- Use to correct bony defects in implants with peri-implantitis

- Utilises glymatrix technology.

To shop the OSSIX product range visit dentsplysirona.com/ossix.on 